\title{
Association of nutritional delivery on skeletal muscle wasting and inflammation in critically ill adult patients: a systematic review
}

\author{
Kaifeng Liang ${ }^{1}$ and Zudin Puthucheary ${ }^{1,2}$ \\ ${ }^{1}$ William Harvey Research Institute, Barts and The London School of Medicine and Dentistry, Queen Mary University \\ of London, EC1M 6BQ and \\ ${ }^{2}$ Adult Critical Care Unit, Royal London Hospital, London, UK
}

Skeletal muscle wasting and weakness is the single greatest contributor of persistent functional disability in critical care survivors. ${ }^{(1,2)}$ Nutrition therapy presents a huge potential in addressing ICU-associated skeletal muscle wasting by targeting excess substrate availability and inflammation. A deeper understanding of the predictive values of metabolic profile and inflammatory phenotype during critical illness will spur a paradigm shift toward individualised treatment. The aim of this review was to systematically examine the association of energy/protein delivery on skeletal muscle mass and inflammatory changes in critically ill adult patients.

The MEDLINE and EMBASE databases were searched for interventional and observational studies of nutrition therapy in critically ill patients aged 18 years or older, published in English up until 26 June 2020. Inclusion criteria were measurements of skeletal muscle mass/volume at 2 or more time points and documentation of the nutrition therapy/feeding protocol used during their stay in the intensive care units. The primary outcome was skeletal muscle mass changes, and the secondary outcome was inflammatory marker changes.

The search yielded 203 results, of which 6 (3 randomised controlled trials and 3 prospective cohort studies) were included in this review. Of the included studies, the population was largely heterogenous with 3 studies taken place in a general ICU, 2 in medical patients, and 1 in head injury patients only. Among the 243 participants in total, the Acute Physiology and Chronic Health Evaluation (APACHE II) on ICU admission varied widely (range 11-33), as did participant age (range 19-90 years), and sex (59\%-100\% male). Participants in the included studies experienced a varying degree of reduction in skeletal muscle mass/volume; median loss were $3-20 \%$ by the first week, $18-20 \%$ by 10 days, and $20-22 \%$ by the second week of ICU admission. Critical illness was associated with an acutely elevated levels of inflammatory mediators, followed by subsequent reduction within 7 days. A variety of methodologies and markers were used to assess skeletal muscle mass and inflammation, which deemed a meta-analysis inappropriate. No clear relationship between energy/protein delivery and skeletal muscle mass changes was identified. Nutrition therapy appeared to have an anti- inflammatory and immune-enhancing effect, observed with low- to moderate- quality evidence.

This systematic review highlighted a lack of high-quality evidence to clearly define the association between energy/protein delivery and skeletal muscle mass changes in critically ill patients. Nutrition therapy appeared to have an anti-inflammatory and immune-enhancing effect, but the true association needs to be further investigated. It also demonstrated the difficulties in interpreting results when there is substantial variation in the methodology and heterogeneity in the patient population. Future research should ideally be performed in adequately powered, unbiased clinical datasets generated in observational studies or randomised controlled trials, with detailed documentation of energy/protein dose, timing, and delivery.

\section{References}

1. Iwashyna, T. J., Ely, E. W., Smith, D. M. \& Langa, K. M. (2010) JAMA - J. Am. Med. Assoc. 304, 1787-1794.

2. Herridge, M. S. et al. (2011) N. Engl. J. Med. 364, 1293-1304. 